\title{
Estudio de los requerimientos de blindaje para aceleradores lineales con haces sin filtro aplanador en modalidad IMRT
}

\author{
Study of shielding requirements for linacs with flattening filter-free \\ beams used in IMRT techniques
}

Revista Investigaciones y Aplicaciones

Nucleares, 5, 54-65, 2021

Recibido: 30 de junio de 2021

Aceptado: 29 de septiembre de 2021

Publicado en línea: 23 de noviembre de 2021

Doi: https://doi.org/10.32685/2590-7468/

invapnuclear.5.2021.587

\section{(c) (1)}

Esta obra está bajo licencia internacional Creative Commons Reconocimiento 4.0.
Angelo Albano Reyes Carvajal ${ }^{1}$, María Esperanza Castellanos ${ }^{2}$

Citación: A. A. Reyes y M. E. Castellanos, "Estudio de los requerimientos de blindaje para aceleradores lineales con haces sin filtro aplanador en modalidad IMRT", Revista Investigaciones y Aplicaciones Nucleares, n. ${ }^{\circ}$ 5, pp. 54-65, 2021. https://doi.org/10.32685/2590-7468/invapnuclear.5.2021.587

\section{Resumen}

En este trabajo se estudiaron los parámetros usados para el cálculo de blindajes en aceleradores lineales (linac) de electrones modernos que pueden operar con o sin filtro aplanador en el haz (haces FF y FFF), en modalidad de radioterapia por intensidad modulada (IMRT). En particular, se analizaron los espesores decirreductores (TVL), la carga de trabajo (W) y los factores de uso (U) de haz primario, con el objetivo de determinar las diferencias tanto en requerimiento de espacio como de costos para un búnker. Se estudiaron las propiedades espectrales de haces FF y FFF de $6 \mathrm{MV}$, a partir de espectros publicados, y su efecto en parámetros como la energía media del haz y los TVL. Se encontró que la energía media en haces FF puede ser 1,15 veces superior a la de haces FFF, mientras que los TVL para haces FFF pueden ser inferiores en un $16 \%$ a los de haces FF, resultados consistentes con datos publicados. Se hizo un estudio de carga de trabajo y de factores de uso de haz primario para dos aceleradores de última generación operados en un centro de alta carga de trabajo en tratamientos con IMRT y arcoterapia de modulación volumétrica (VMAT), con el objetivo de realizar cálculos de blindaje representativos para un centro moderno de radioterapia. Con un ejemplo de búnker, se halló que el espesor de las barreras primarias para un linac operado con haces FFF puede ser inferior hasta en un $19 \%$ al requerido para haces FF; también, se reduce el espesor de las barreras secundarias necesarias, lo cual se explica en parte con la disminución de la carga de trabajo de radiación de fuga, $W_{L}$, en haces FFF de un $54 \%$, como consecuencia de la disminución del número de unidades de monitor (UM) requerido para

${ }^{1}$ Clínica San Rafael, Pereira, Colombia.

${ }^{2}$ Pontificia Universidad Javeriana, Bogotá, Colombia.

Autor de correspondencia: Angelo Albano Reyes Carvajal, ar.carvajal@hotmail.com 
un tratamiento con haces FFF, en comparación con haces FF. Cuando se utilizan haces combinados, las ventajas de blindaje se ven reducidas dependiendo de la combinación utilizada; por ejemplo, se estableció que al aumentar el modo de operación de un linac de un $20 \%$ a un $40 \%$ con haz FFF, la ventaja en reducción de blindaje puede llegar hasta $9 \%$ para barreras primarias y secundarias. Mediante factores de uso de haz primario, correspondiente a los modos de operación de un linac moderno, como los estudiados, y en combinación con haces únicos FFF, el espesor de las barreras primarias y secundarias se puede reducir hasta en un $30 \%$, optimizando así costos y espacios, sin sacrificar la protección del público y trabajadores. Por último, como consecuencias prácticas de la reducción de espesores de blindaje, es posible reducir entre $13 \%$ y $17 \%$ la cantidad de concreto ordinario para la construcción de un búnker como el del ejemplo analizado, y se puede llegar a un ahorro específico en espacio entre $6 \mathrm{~m}^{2}$ a $11,8 \mathrm{~m}^{2}$.

Palabras clave: filtro aplanador, TVL, carga de trabajo, radiación de fuga, factor de uso.

\section{Abstract}

An analysis was performed of the parameters used in shielding calculations for modern electron linear accelerators (linacs) that can operate with or without a flattening filter in the beam (FF and FFF) in the intensity modulated radiotherapy (IMRT) modality. In particular, the tenth-value layer (TVL), workload (W) and primary beam use factor (U) were analyzed to determine the differences in both space requirements and costs for a bunker. The spectral properties of 6-MV FF and FFF beams were determined from published spectra and the effect of these properties on parameters, such as the mean beam energy and TVLs, were studied. The average energy for FF beams was found to be 1.15 times higher than that of FFF beams, whereas the TVLs for FFF beams were found to be $16 \%$ lower than those for FF beams, which is consistent with published data. The workload and primary beam use factors were investigated for two last-generation accelerators operated in a high-workload center for treatments with IMRT and volumetric modulated arc therapy (VMAT), with the objective of performing shielding calculations representative of a modern radiotherapy center. For the case of a bunker, a linac operated using FFF beams requires up to $19 \%$ thinner primary barriers than using FF beams, as well as thinner secondary barriers, partly because of a $54 \%$ decrease in the workload of leakage radiation, $\mathrm{W}_{\mathrm{L}}$, for FFF beams as a consequence of the decrease in the number of required monitor units (MU) compared to FF beams. The use of combined beams decreases shielding benefits, where the extent of reduction depends on the combination used: for example, increasing the operating mode of a linac from $20 \%$ to $40 \%$ with an FFF beam can reduce shielding for primary and secondary barriers by up to $9 \%$. Primary beam use factors for modern linac modes of operation, such as those studied, and in combination with single FFF beams can reduce the thickness of primary and secondary barriers by up to $30 \%$, thus optimizing costs and space without sacrificing protection of the public and workers. Finally, the practical consequences of thinner shielding are a reduction of between $13 \%$ and $17 \%$ in the quantity of ordinary concrete required to build the considered bunker and space savings between $6 \mathrm{~m}^{2}$ and $11.8 \mathrm{~m}^{2}$.

Keywords: flattening filter, TVL, workload, leakage radiation, use factor. 


\section{Introducción}

os aceleradores lineales (linear accelerator, linac) convencionales utilizados en radioterapia están equipados con un filtro aplanador (flattening filter, FF), cuyo objetivo es producir una distribución de dosis uniforme en el campo, considerando un medio homogéneo. Los avances tecnológicos han permitido una evolución de las técnicas de tratamiento con radioterapia externa, desde la técnica convencional o bidimensional (2D), pasando por la conformal tridimensional (3DCRT, por su sigla en inglés), hasta la radioterapia de intensidad modulada (IMRT, por su sigla en inglés), y recientemente la arcoterapia de modulación volumétrica (VMAT, por su sigla en inglés), con las cuales se administran tratamientos de intensidad modulada de manera más eficiente. Todas estas técnicas se han desarrollado con equipos que disponen de filtro aplanador. Sin embargo, investigaciones recientes han demostrado que en la práctica moderna de radioterapia con IMRT, VMAT o con haces pequeños no es indispensable la presencia del filtro en el haz, puesto que este se modula antes de la llegada al paciente, produciendo haces inherentemente no planos, o el haz pequeño es por su naturaleza relativamente plano. Así, en los linac modernos se cuenta también con la opción de haces sin filtro aplanador, o haces FFF (flattening filter free) [1], [2].

La inclusión o no del filtro aplanador implica diferencias en las características del haz en cuanto a su calidad, intensidad y distribución lateral de dosis, principalmente. El haz FFF es menos energético, particularmente en el centro, por cuanto el filtro aplanador endurece el haz (filtra los haces de energías bajas), lo cual ocurre cuando el haz de electrones usado en los haces FFF es el mismo que en los FF, como es el caso de los linac Varian [3]; esto ha sido analizado en diferentes trabajos publicados. Lind [4] encontró que la calidad del haz $\left(T P R_{10}^{20}\right)$ para un haz de 6 MV FFF puede ser de 0,664 , mientras que para un haz de $6 \mathrm{MVFF}$ es de 0,681; Vassiliev [5] halló que el porcentaje de dosis en profundidad (PDD) en agua para un haz de $6 \mathrm{MVFFF}$ es igual al de un haz de $4 \mathrm{MVFF}$, datos consistentes con los publicados por el IPEM (Institute of Physics and Engineering in Medicine) [3]. Por otra parte, Vassiliev y el IPEM reportaron una tasa de dosis superior en un factor de 2,1 y 2,3 en el eje en haces FFF de $6 \mathrm{MV}$, en comparación con haces FF [3], [6].
En otro enfoque, la energía de los electrones incidentes en el blanco se aumenta para tratar de hacer coincidir la calidad de los haces FFF a los convencionales (FF), pero de acuerdo con el IPEM [3], por cuanto no se trata simplemente poner y quitar el filtro, hay diferencias irreconciliables en el espectro del haz que pueden influir, por ejemplo, en las componentes de radiación dispersa.

Como consecuencia de las diferencias mencionadas y del uso más extendido de linac con haces FFF, los parámetros utilizados en el cálculo de blindajes como los espesores decirreductores (TVL) y las fracciones de dispersión para haces FF, no son adecuados para haces FFF. Por consiguiente, y con la visión de que a futuro los haces FFF se establecerán como estándar en la práctica clínica, es importante estudiar cómo cambian los parámetros de blindaje para haces FFF en comparación con haces FF, y determinar cuál es su impacto en el diseño de un búnker. Dado que, de acuerdo con el IPEM [3], hasta el año 2016 no había haces de 18 MV FFF disponibles en el mercado, y a que se observa una tendencia a preferir los haces de $6 \mathrm{MV}$ para los tratamientos con haces modulados en intensidad, en el presente trabajo se estudiaron haces filtrados y sin filtro de $6 \mathrm{MV}$ : se analizaron las diferencias espectrales, se determinaron los TVL para haces FFF y con los resultados se calcularon los espesores necesarios de material blindante para un ejemplo de un búnker en particular. Estos resultados se compararon con los espesores obtenidos para haces FF y se establecieron las consecuencias prácticas en requerimiento de espacio y de costos. Además, se estudió la influencia en ese diseño de la carga de trabajo y del factor de uso de haz primario en el cálculo de blindaje, para técnicas VMAT e IMRT.

\section{Materiales y métodos}

\subsection{Estudio de diferencias espectrales de haces FF y FFF}

Las diferencias espectrales se evaluaron a través de la energía medida $(\bar{E})$ para haces de $6 \mathrm{MV}$ con filtro y sin filtro aplanador. La energía media de un haz de fotones se puede calcular mediante la ecuación 1 [7]; donde $\Phi_{E}$ es la fluencia diferencial en energía de fotones. El numerador representa la fluencia energética y el denominador, la fluencia total de fotones producidos en el rango desde cero hasta la máxima energía. 


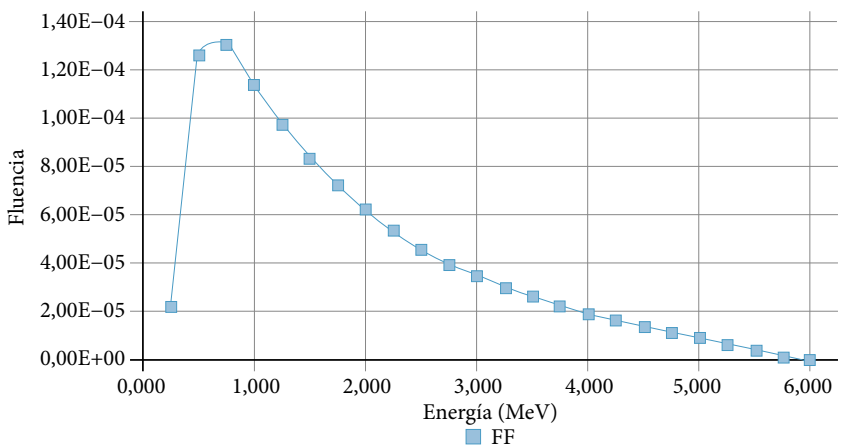

Figura 1. Espectro sobre el eje de un haz de $6 \mathrm{MV}$ (Varian) con filtro aplanador publicado por Rogers y Sheikh [8]

$$
\bar{E}_{\Phi}=\frac{\int_{0}^{E \max } E \Phi_{E} d E}{\int_{0}^{E m a x} \Phi_{E} d E}
$$

Se utilizó el espectro con filtro de una máquina Varian publicado por Rogers y Sheikh [8] (figura 1) para obtener un espectro de haz de $6 \mathrm{MVFFF}$, lo cual se realizó en dos etapas. Primero, se calculó el espectro del haz incidente en el filtro aplanador a partir de la ecuación de atenuación simple (ecuación 2, [7]), considerando el espesor central de un filtro aplanador para esta clase de máquinas. Posteriormente, por cuanto en los haces FFF se reemplaza el filtro aplanador por una placa plana de espesor inferior o igual a $2 \mathrm{~mm}$, fabricada de diversos materiales, para filtrar electrones y fotones de baja energía [1], se utilizó nuevamente la ecuación de atenuación simple para obtener el espectro del haz después de atravesar una placa plana de cobre de 0,8 mm [1], [4], [5]. Para los espectros con y sin filtración, finalmente se determinaron las $\Phi_{E}$ necesarias para el cálculo de la $\bar{E}$.

$$
\frac{I}{I_{0}}=e^{-\mu L}
$$

$I_{o}$ corresponde al número de fotones incidentes en el filtro por cada bin de energía; I es el número de fotones que atraviesan el filtro; $\mu$ es el coeficiente de atenuación [9] y $L$ es el espesor del filtro en el centro.

\subsection{Determinación de TVL para haces FFF}

Los TVL para haces FFF fueron calculados usando un modelo desarrollado por LaRiviere y Nelson [10], el cual permite determinar el espesor de material blindante necesario para reducir la dosis absorbida a un porcentaje dado. El modelo establece que la transmisión de fotones a través de un material puede escribirse en términos de una integral sobre el espectro de energía del haz. Es decir, la dosis absorbida en el aire en el seno de aire para un espesor $t$ se establece a partir de la ecuación 3 [10], donde $\mu$ es el coeficiente lineal de atenuación, $\Phi$ es la fluencia de partículas, $B$ es el factor de acumulación buildup de la dosis absorbida, $\left(\frac{\mu_{e n}}{\rho}\right)_{\text {air }}$ es el coeficiente de absorción másico de energía para el aire.

$$
D(t)=\int_{E \min }^{E \max } E \varphi(E)\left(\frac{\mu_{e n}(E)}{\rho}\right)_{\text {air }} B(E, \mu t) e^{-\mu(E) t} d E
$$

El factor de acumulación buildup de dosis absorbida se obtiene de la expresión de Berger en la ecuación 4 [10], donde los parámetros $\alpha(E)$ y $\beta(E)$ se toman de Trubey [11], y los coeficientes de atenuación para el material blindante y el coeficiente de absorción de energía en aire del National Institute of Standards and Technology (NIST) [9].

$$
B(E, \mu t)=1+\alpha(E) \mu t e^{-\mu t \beta(E)}
$$

Con el modelo anterior, los espesores decirreductores $\mathrm{TVL}_{1}$ se obtuvieron de la transmisión definida por la relación $\frac{D(t)}{D(o)}=0,1$ [10]; así el $\mathrm{TVL}_{1}$ es el espesor necesario para atenuar el haz en un factor de 10 . El TVL $\mathrm{TV}_{2}$ es el espesor necesario para atenuar el haz en un factor adicional de $10 \mathrm{y}$, finalmente, el $\mathrm{TVL}_{3}$ tomado en esta investigación como TVLe, es un espesor adicional más para atenuar el haz en otro factor de 10 [12].

Para validar el modelo, se tomaron los espectros publicados por Sheikh-Bagheri y Rogers [8] para haces de $6 \mathrm{MV}$ y $10 \mathrm{MV}$ con filtro aplanador y se calcularon los $\mathrm{TVL}_{1}$ y los TVLe, los cuales se compararon con los reportados en el NCRP151 [13]. Posteriormente se aplicó el modelo validado para obtener los TVL de haces 6MVFFF a partir del espectro FFF encontrado en este estudio. Los resultados se comparan con los publicados por Kry, Mohan y Vassiliev [14].

\subsection{Estudio de carga de trabajo $(\mathrm{W})$}

Se estudió la carga de trabajo de dos unidades de tratamiento de un centro de radioterapia moderno de alta ocupación, en el cual los pacientes son tratados con haces FF de $6 \mathrm{MV}$. La información obtenida permite un análisis del tipo de tratamientos realizados, lo cual es sustancial para determinar 
la cantidad de unidades monitor en promedio por paciente, requerido para el cálculo de la carga de trabajo. No se abordó en este estudio el posible impacto en la carga de trabajo de los esquemas de tratamiento hipofraccionados.

El trabajo consistió en obtener los datos de pacientes tratados en cinco días diferentes de alta ocupación, e identificar la dosis prescita, la profundidad de tratamiento, la técnica usada y el número de UM en cada una de las máquinas. A su vez, para establecer la relación entre el número de UM relacionado con la calibración del haz (UM para una cierta dosis a la profundidad máxima ionización a $1 \mathrm{~m}$ de la fuente) y las administradas en los tratamientos con haz modulado en intensidad, según las dosis prescritas, se calcularon los planes de tratamiento en 3DCRT.

Para los mismos casos se realizaron cálculos en IMRT o VMAT y se determinó el factor $C_{I}$ (ecuación 5), el cual considera el aumento del número de UM en IMRT en comparación con 3DCRT; este factor es necesario para los cálculos de la carga de trabajo de radiación de fuga $\left(W_{L}\right)$ para el haz FF.

$$
C_{I}=\frac{M U_{I M R T}}{M U_{c o n v}}
$$

Como el NCRP151 [13] no considera el caso de haces FFF, y debido a que estudios posteriores a la publicación de esa recomendación muestran que cuando se utilizan haces sin filtración el factor de corrección de la carga de trabajo de fuga cambia, en este estudio se utiliza el enfoque propuesto por Kry, Mohan y Vassiliev [14], para la corrección de este parámetro, usando el factor calculado mediante la ecuación 6. Este factor de corrección $\left(C_{I F}\right)$ tiene en cuenta el aumento de las UM debido a la técnica VMAT utilizada (primer término), la diferencia en el número de UM que se requieren para administrar los tratamientos con y sin filtración (segundo término) y el tercer término tiene en cuenta que se requiere una corriente de electrones diferente para producir 1 UM cuando se elimina el filtro.

$$
C_{I F}=\left(\frac{M U_{F F I M R T}}{M U_{F F c o n v}}\right)\left(\frac{M U_{\text {treatmentFFF }}}{M U_{\text {treatmentFF }}}\right)\left(\frac{\text { Target }_{\text {current }}}{\text { FFF }}\right)
$$

En la ecuación 6 los términos dos y tres fueron extraídos de estudios realizados por Vassiliev [15], y Titt, Vassiliev y Pönisch [16], respectivamente.

\subsection{Factor de uso (U) para IMRT y VMAT (haz primario)}

Para el cálculo del factor de uso de haz primario en cada máquina, se analizó el total de tratamientos realizados en una semana y se determinó la dosis (cGy) acumulada por el haz en cada posición del gantry en ángulos de $1^{\circ}$. Esta dosis acumulada se dividió por la dosis total de tratamiento semanal, con lo cual se obtuvo la fracción de carga de trabajo de haz primario que se dirige hacia una barrera primaria. Específicamente, para la técnica VMAT, conociendo el ángulo de inicio y parada junto con la direccionalidad, se asumió que, para cada ángulo comprendido dentro del arco, la dosis administrada se distribuía en partes iguales.

\subsection{Cálculo de barreras primarias y secundarias para haces FF y FFF}

En la figura 2 se presenta el diseño de búnker utilizado para determinar las barreras primarias y secundarias en este estudio. El plano fue adecuado del ejemplo presentado en la sección 7 del informe 151 del NCRP [13].

Se calcularon espesores en concreto ordinario $\left(\rho=2,35 \mathrm{~g} / \mathrm{cm}^{3}\right)$, material ampliamente usado, ya que puede verterse en variadas configuraciones y provee un buen blindaje y fuerza estructural.

El enfoque que se presenta para evaluar los espesores de blindaje requeridos es el siguiente:

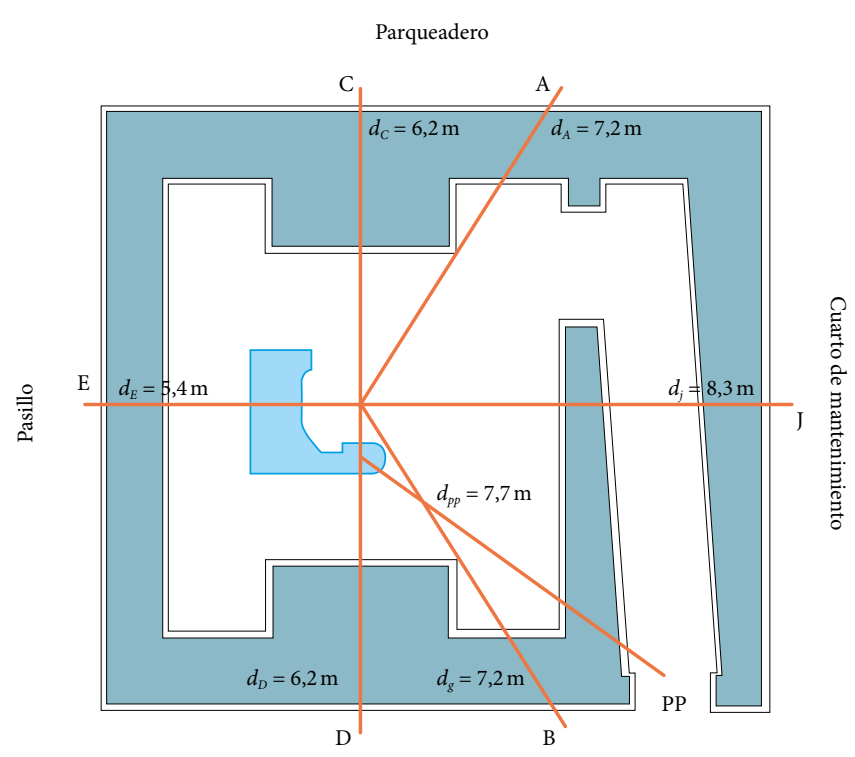

Cuarto de control de tratamiento

Figura 2. Plano de búnker utilizado para cálculos de blindaje Esquema general tomado del NCRP151 [13] 
Haz con filtración. Se utiliza el escenario de dedicación exclusiva del acelerador a técnica VMAT, con haz de $6 \mathrm{MV}$ FF. Se halla la carga de trabajo (W), el promedio de pacientes tratados en una hora $\left(\mathrm{N}_{\mathrm{h}}\right)$ y el número máximo de pacientes tratados en una hora $\left(\mathrm{N}_{\text {max. }}\right)$. Los resultados para la máquina 1 en este estudio se usan para el cálculo. Los demás parámetros como TVL, fracciones de dispersión, factor de uso, entre otros, necesarios para los cálculos, fueron tomados del NCRP. exclusiva a técnica VMAT, con haz de 6 MV FFF. Se aplican los mismos factores: carga de trabajo (primaria y dispersa), $\mathrm{N}_{\text {máx. }}$ y $\mathrm{N}_{\mathrm{h}}$. Los TVL necesarios para la barrera primaria y $W_{L}$ se toman de los resultados de este estudio. Las fracciones de dispersión y los TVL en concreto para radiación dispersa por el paciente se adoptan de los reportados por Kry, Mohan y Vassiliev [14]; para que los resultados sean comparables entre haces con y sin filtro, el factor de uso utilizado es el reportado en el NCRP151.

Haces combinados. Para evaluar el impacto del uso combinado se suponen dos aproximaciones. La primera considera los resultados reportados por Mishra y Palani [17], quienes establecen que en centros donde se utilizan haces combinados de $6 \mathrm{MV}$, el $80 \%$ de los pacientes se tratan en modo FF y el $20 \%$ restante en modo FFF. Además, teniendo en cuenta que cada vez más se recurre a técnicas de tratamiento como la radioterapia estereotáctica corporal (que administra con alta precisión una dosis alta de radiación a blancos pequeños y bien definidos, mediante haces FFF en máquinas modernas) [18], se ha establecido una segunda aproximación, asumiendo que el $60 \%$ de los pacientes se tratan con haces FF y el $40 \%$ con haces FFF.

Además de los anteriores enfoques, se evalúan los cálculos de blindaje para los escenarios presentados, utilizando los factores de uso encontrados en este estudio. Lo anterior, además, para analizar el hecho de que en técnicas VMAT e IMRT no existen angulaciones preferenciales del gantry o brazo del linac.

Para identificar las consecuencias prácticas tanto en requerimientos de espacio y costos, se establece el porcentaje de ahorro de área con los nuevos espesores y la cantidad de metros cúbicos de concreto ordinario, para cada uno de los escenarios utilizados.

\section{Resultados y discusión}

\subsection{Diferencias espectrales de haces FF y FFF}

En la tabla 1 se presenta la energía media de los haces estudiados; se constata que los haces filtrados son más energéticos: para haces $6 \mathrm{MV}$ filtrados, la $\bar{E}$ es 1,55 veces mayor que para el haz no filtrado. Esta diferencia implica que la calidad de haz en haces FF y FFF difiera para un mismo potencial nominal.

Tabla 1. Energía $\bar{E}$ para haces FF y FFF de $6 \mathrm{MV}$

\begin{tabular}{cc}
\hline Haz & $\bar{E}(\mathbf{M e V})$ \\
\hline $6 \mathrm{FF}$ & 1,80 \\
$6 \mathrm{FFF}$ & 1,56 \\
\hline
\end{tabular}

Para visualizar la diferencia espectral entre haces filtrados y no filtrados, en la figura 3 se ilustra el espectro del haz FFF obtenido para un haz de $6 \mathrm{MV}$ a partir del espectro de Roger y Sheikh [8]. Se observa, también, que el máximo de emisión en el espectro para el haz FFF (normalizado) se desplaza hacia la izquierda, en comparación con el haz FF, lo cual se va a reflejar en la calidad del haz evaluada en la distribución de dosis en un medio homogéneo. Las variaciones observadas en la distribución energética del haz se verán reflejadas, igualmente, en los parámetros usados para el cálculo de blindajes como los TVL.

\subsection{Determinación del TVL para haces FFF}

En la tabla 2 se detallan los TVL encontrados para haces de $6 \mathrm{MV}$; con estos resultados se valida el modelo aplicado que se describe en la sección 2.2. Al compararlos con los reportados en el NCRP 151, se encuentra que para el haz de $6 \mathrm{MV}$ el modelo aplicado no hay diferencia en el $\mathrm{TVL}_{1}$ y mientras que para el TVLe la diferencia es de $6 \%$. El método se aplicó también al espectro de un haz de $10 \mathrm{MV}$; aquí se hallaron diferencias de $2 \%$ y $0 \%$, respectivamente. Lo anterior indica que el modelo aplicado para determinar los TVL es adecuado. Las diferencias en los $\mathrm{TVL}_{1}$ y TVLe se deben principalmente al error que presentan los coeficientes de Berger [9] y a los espectros utilizados. 

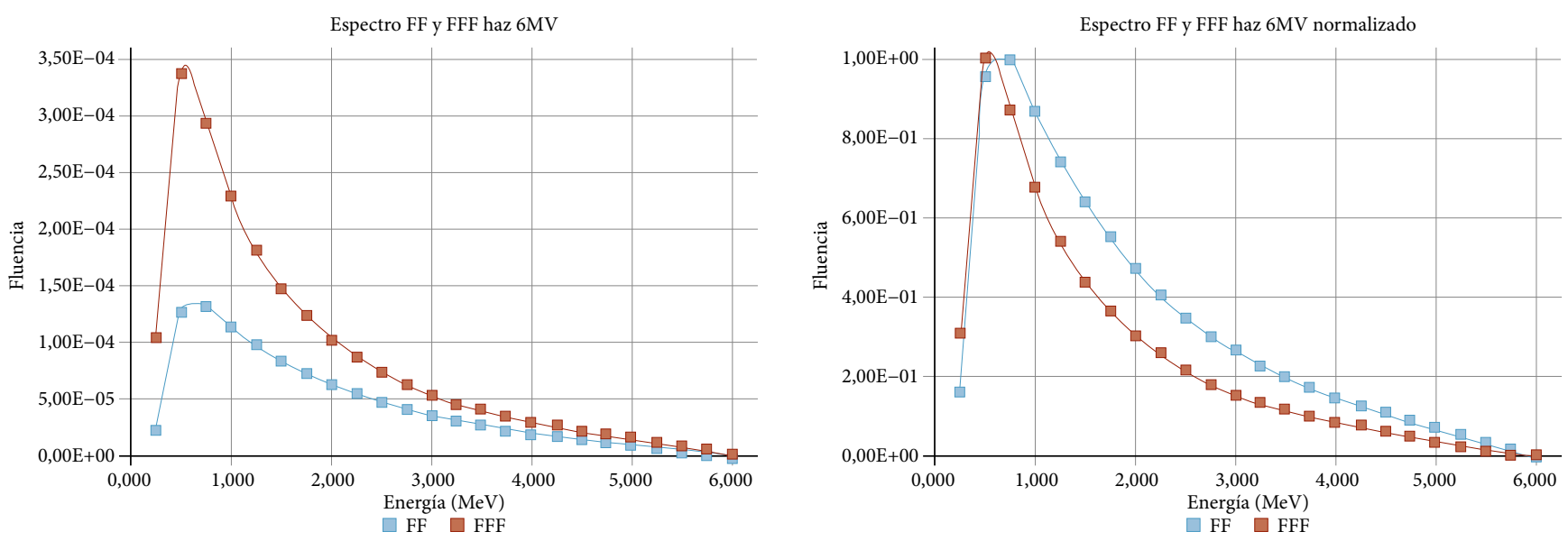

Figura 3. Espectro encontrado para el haz FFF de $6 \mathrm{MV}$ a partir del espectro FF de Rogers y Sheikh [8]

Izquierda: espectros sin normalizar; derecha: espectros normalizados.

Tabla 2. $\mathrm{TVL}_{1}$ y TVLe para haces de $6 \mathrm{MV}$ y $10 \mathrm{MV}$ con filtro aplanador, para concreto ordinario

\begin{tabular}{cccc}
\hline Referencia & Haz $\mathbf{( M V )}$ & $\mathbf{T V L}_{\mathbf{1}} \mathbf{( c m )}$ & $\mathbf{T V L}_{\mathbf{e}} \mathbf{( c m )}$ \\
\hline Modelo aplicado & $6 \mathrm{FF}$ & 37 & 31 \\
NCRP & $6 \mathrm{FF}$ & 37 & 33 \\
Modelo aplicado & $10 \mathrm{FF}$ & 42 & 37 \\
NCRP & $10 \mathrm{FF}$ & 41 & 37 \\
\hline
\end{tabular}

Para el haz de $6 \mathrm{MV}$ sin filtración (haz FFF de 6MV), los TVL hallados a través del modelo planteado se presentan en la tabla 3. Al compararlos con los resultados de Kry, Mohan y Vassiliev [14], la diferencia máxima encontrada fue del $4 \%$, por consiguiente, se comprueba la validez del modelo y el cálculo de los TVL para el haz de $6 \mathrm{MV}$.

Tabla 3. $\mathrm{TVL}_{1}$ y TVLe para el haz de $6 \mathrm{MV}$ sin filtro aplanador

\begin{tabular}{cccc}
\hline Referencia & Haz (MV) & TVL $_{\mathbf{1}}(\mathbf{c m})$ & TVL $_{\mathbf{e}}(\mathbf{c m})$ \\
\hline Modelo aplicado & $6 \mathrm{FFF}$ & 31 & 28 \\
Kry, Mohan y Vassiliev & $6 \mathrm{FFF}$ & 30 & 27 \\
\hline
\end{tabular}

Los valores de los TVL para haces sin filtro aplanador son un $16 \%$ más pequeños que los reportados por el NCRP151.

\subsection{Carga de trabajo}

La carga de trabajo para el haz de $6 \mathrm{MV}$ con filtración se determinó con datos reales de un centro oncológico moderno para dos máquinas de tratamiento operadas solamente en modo FF. Para la primera máquina analizada, se determinó que el $98 \%$ de los pacientes son tratados con la técnica VMAT, y para la máquina 2, la técnica de tratamiento es $15 \%$ convencional y $85 \%$ es VMAT e IMRT (DMLC y step and shoot).

Los tratamientos analizados para la carga de trabajo en la máquina 1 se muestran en la figura 4; en general, fueron tratamientos de próstata con un $63 \%$, cabeza y cuello $23 \%$, radiocirugía $8 \%$, entre otros. De igual forma, en la figura 5 se presentan los tratamientos analizados para la carga de trabajo de la máquina 2, donde el $34 \%$ corresponde a próstata, $24 \%$ a seno, $10 \%$ cabeza y cuello, $8 \%$ recto-colon y otros.

Tratamientos (total 275) Máquina 1

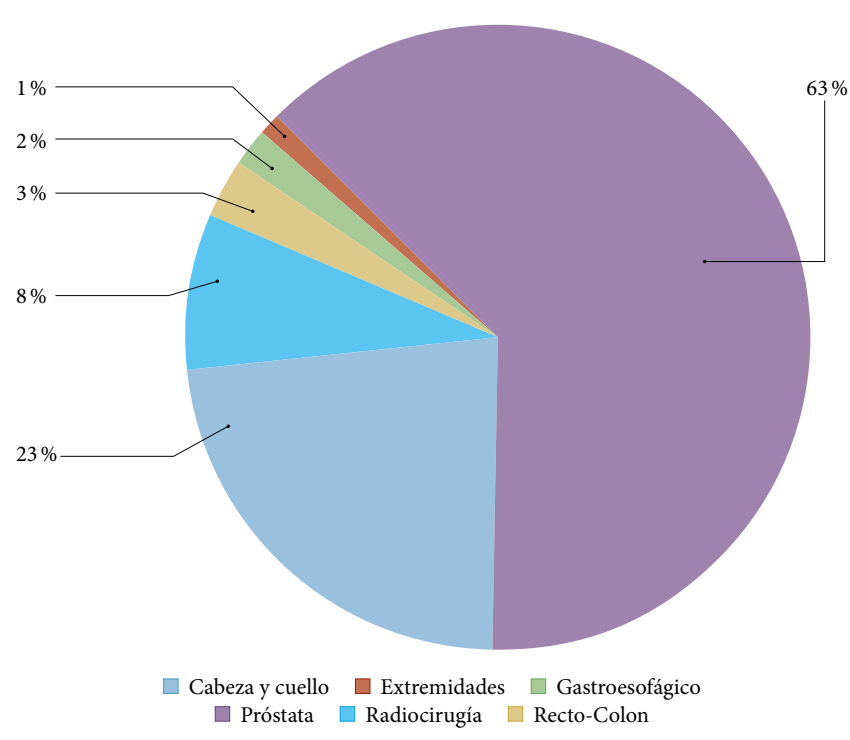

Figura 4. Máquina 1: tratamientos analizados para carga de trabajo y factor de uso Información correspondiente a 5 días de tratamientos en un centro oncológico de alta ocupación 


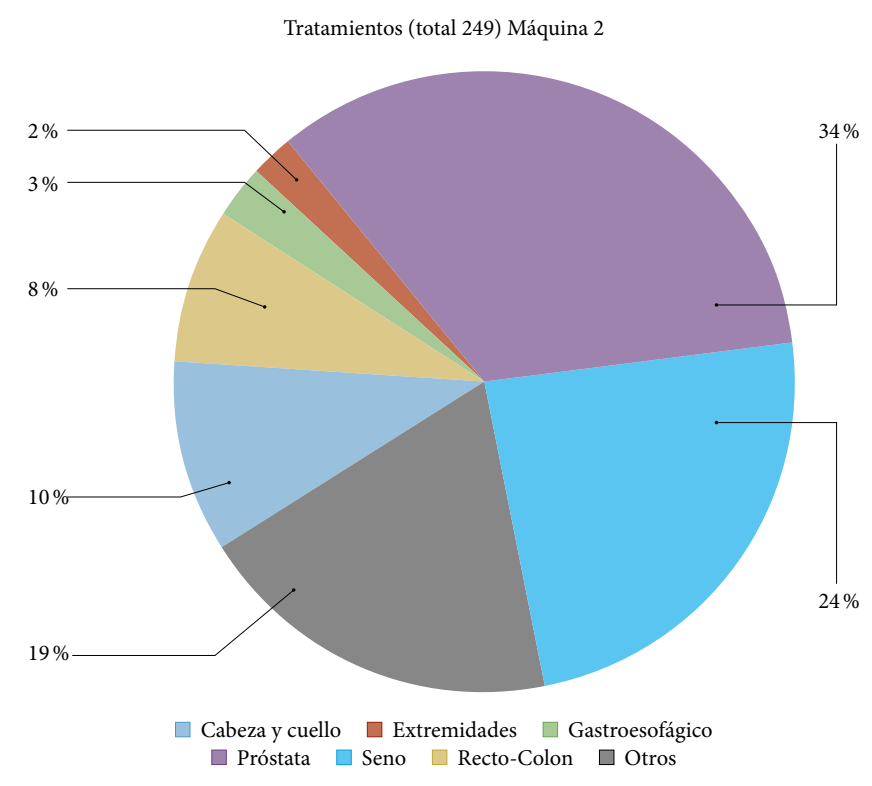

Figura 5. Máquina 2: tratamientos analizados para carga de trabajo y factor de uso

Información correspondiente a 5 días de tratamientos en un centro oncológico de alta ocupación

En la tabla 4, se presenta la dosis prescrita por semana en cada máquina y la carga de trabajo calculada, considerando la profundidad media de tratamiento. El factor promedio encontrado para calcular la carga de trabajo, considerando dosis en la profundidad de máxima ionización, a partir de la dosis prescrita, hallado para los tratamientos de próstata, cabeza y cuello, seno, extremidades y demás de las figuras 4 y 5 , fue 1,3 .

Tabla 4. Dosis prescrita en una semana para máquinas 1 y 2

\begin{tabular}{ccc}
\hline Máquina & Dosis (Gy/semana) & W (Gy/semana) \\
\hline 1 & 583 & 758 \\
2 & 645 & 839 \\
\hline
\end{tabular}

Por otra parte, utilizando los tratamientos analizados en las dos máquinas, se encontró que el factor $C_{I}$ necesario para determinar la carga de trabajo de radiación de fuga para el haz FF en las dos máquinas, es en promedio de 3,1. Siendo más restrictivos, para los cálculos se trabajó un factor de 3,7, el mayor valor encontrado.

Para la $W_{L}$ (carga de trabajo de radiación de fuga) del haz FFF, se utilizaron los factores publicados por Vassiliev [15], y Titt, Vassiliev y Pönisch [16], a saber: 3,7, 0,5 y 0,9 (tabla 5), obteniendo un valor un factor $C_{I F}$ (ecuación 6) de 1,7.
Tabla 5. Valores de los términos para la ecuación 6

\begin{tabular}{cc}
\hline Descripción & Valor \\
\hline Primer término & 3,7 \\
Segundo término & 0,5 \\
Tercer término & 0,9 \\
\hline
\end{tabular}

A partir del resultado anterior, se calculó la carga de trabajo para la radiación de fuga en haces con y sin filtro aplanador, que se presenta en la tabla 6; allí se observa que, al eliminar el filtro aplanador, la carga de trabajo de radiación de fuga disminuye hasta en un $54 \%$, debido principalmente a que el factor $C_{I F}$ fue inferior al factor $C_{I}$. Este comportamiento de la $W_{L}$ se debe a que la cantidad de UM requeridas para un tratamiento con haz FFF, es menor que para un tratamiento con haz FF; y además, a que la corriente blanco para un tratamiento con haz sin filtración es menor que para uno con filtración [14].

Tabla 6. Carga de trabajo de radiación de fuga en Gy/semana para haz FF y FFF de $6 \mathrm{MV}$

\begin{tabular}{ccc}
\hline Haz & $\begin{array}{c}\mathbf{W}_{\mathrm{L}} \\
\text { Máquina 1 }\end{array}$ & $\begin{array}{c}\mathbf{W}_{\mathrm{L}} \\
\text { Máquina 2 }\end{array}$ \\
\hline $6 \mathrm{FF}$ & 2806 & 2757 \\
$6 \mathrm{FFF}$ & 1289 & 1336 \\
\hline
\end{tabular}

La reducción porcentual de la $W_{L}$ reportada en este estudio es consecuente con los resultados publicados por Cashmore [19], quien estimó una reducción del 58\%. Además, del estudio realizado de la carga de trabajo, se obtuvo que para las dos máquinas $\mathrm{N}_{\mathrm{h}}$ es 5 y $\mathrm{N}_{\text {máx. }}$ es 8 .

\subsection{Factor de uso (U) VMAT e IMRT (haz primario)}

El factor de uso encontrado para cada uno de los linac estudiados se muestran en las figuras 6 y 7.

Al comparar el resultado del factor de uso VMAT (figura 6) en la máquina 1 , con el factor de uso convencional, se observa que la carga de trabajo se distribuye casi de manera homogénea sobre las barreras primarias. Se nota una pequeña tendencia del factor de uso hacia los $90^{\circ}$, lo cual se debe a que en esta máquina es utilizada para procedimientos de radiocirugía con el haz en esa dirección. Además, el valor máximo de $U$ se presenta también a los $90^{\circ}$ con un valor de $0,47 \%$ aproximadamente. Este resultado, que es inferior al utilizado en el convencional, conlleva reevaluar el factor de uso en los cálculos de blindaje según las técnicas de tratamiento para las cuales es proyectada una máquina. En este 


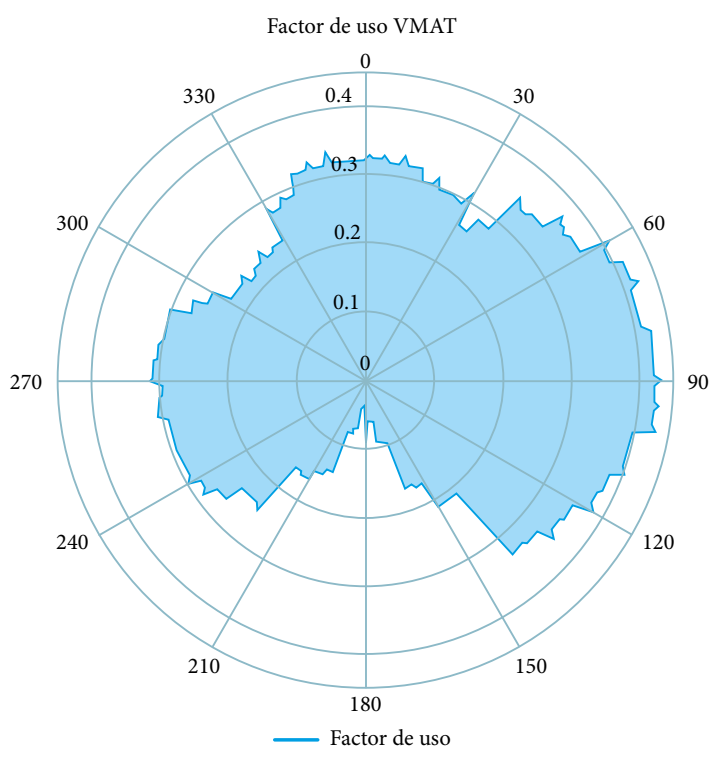

Figura 6. Factor de uso, equipo dedicado a técnica VMAT

estudio se utilizarán tanto los factores de uso convencionales reportados en el NCRP y como los factores de uso aquí encontrados (específicamente en de la máquina 1) para los cálculos de blindajes.

En la figura 7, correspondiente al factor de uso de la máquina 2, donde las técnicas de tratamiento fueron $85 \%$ VMAT e IMRT (DMLC y step and shoot) y $15 \%$ convencional, se observa que los valores del factor de uso del haz primario (U) difieren con los convencionales presentados en el NCRP151. El uso del equipo principalmente está destinado a técnicas IMRT y el valor máximo de U es un $4 \%$ aproximadamente, presentándose a un ángulo de gantry de $180^{\circ}$. Otro aspecto que se analiza es que la distribución de la carga de trabajo sobre las barreras primarias registra tendencia hacia ciertos ángulos $\left(0^{\circ}, 51^{\circ}, 102^{\circ}, 153^{\circ}, 204^{\circ}, 255^{\circ}\right.$ y $\left.306^{\circ}\right)$, que corresponden a las angulaciones utilizadas en tratamientos de próstata, seno y otros en IMRT.

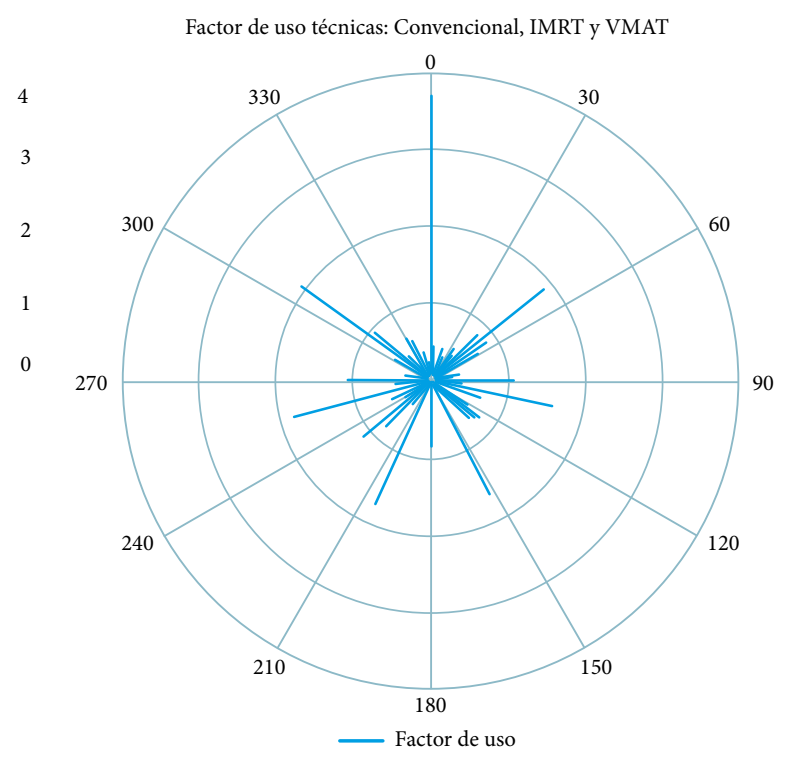

Figura 7. Factor de uso, equipo dedicado a técnicas: convencional, IMRT y VMAT

\subsection{Cálculo de barreras primarias y secundarias para haces FF y FFF}

Al comparar los resultados de los espesores de las barreras del ejemplo del búnker para los dos primeros escenarios descritos en la metodología, es decir, para cuando se asume dedicación exclusiva de la máquina 1 a técnica VMAT con haces FF y FFF y utilizando el factor de uso convencional, se observa que los requisitos de blindaje para las barreras primarias se redujeron hasta en un $19 \%$ para el haz FFF, como consecuencia de un TVL inferior. Estos primeros resultados se detallan en la tabla 7.

Los escenarios considerados corresponden al uso exclusivo del acelerador con haces de $6 \mathrm{MV}$ con y sin filtro aplanador.

Asimismo, para las barreras secundarias la reducción máxima del espesor fue de un $13 \%$. Este resultado no solo se debe a la disminución de las TVL de la radiación dispersa por el paciente, como lo demuestran Kry, Mohan, y Vassiliev [14] en su estudio, sino también, a la disminución de hasta

Tabla 7. Resultados de requerimientos de blindajes para haces con y sin filtro aplanador en $\mathrm{cm}$

\begin{tabular}{|c|c|c|c|c|}
\hline Ubicación & Barrera & Espesor (FF) & Espesor (FFF) & Porcentaje de reducción (\%) \\
\hline C (Parqueadero) & Primaria & 151 & 123 & 19 \\
\hline A (Parqueadero) & Secundaria & 79 & 69 & 13 \\
\hline D (Cuarto control trat.) & Primaria & 159 & 130 & 18 \\
\hline B (Cuarto control trat.) & Secundaria & 95 & 85 & 11 \\
\hline G (Techo) & Primaria & 164 & 134 & 18 \\
\hline H (Techo) & Secundaria & 94 & 84 & 11 \\
\hline L (Laberinto) & Secundaria & 71 & 64 & 10 \\
\hline E (Pasillo) & Secundaria & 92 & 82 & 11 \\
\hline $\mathrm{J}$ (Cuarto mantenimiento) & Secundaria & 81 & 71 & 12 \\
\hline
\end{tabular}


en un $54 \%$ de la carga de fuga $W_{L}$. Los anteriores resultados son congruentes con los reportados por Kry, Mohan, y Vassiliev [14], y Mishra y Palani [17], quienes reportan que las paredes son más delgadas hasta en un $20 \%$ en barreras primarias, y 10-19\% en barreras secundarias.

En este estudio, se supone que la energía de aceleración de los electrones no se alteró cuando se eliminó el filtro aplanador, dando como resultado la disminución de la calidad del haz y, por tanto, una disminución esperada en los requisitos de blindaje. En este caso, puede establecerse que la ventaja de blindaje en modo FFF es del $10 \%$ al $19 \%$.

Cuando se tienen haces combinados con proporciones de $80 \%$ FF y $20 \%$ FFF, se obtiene disminución de hasta un $3 \%$ y $4 \%$ para barreras primarias y secundarias, respectivamente, haciendo que la ventaja de blindaje sea modesta en comparación con la dedicación exclusiva a tratamientos con haces FFF. Esto se debe principalmente a que solo un $20 \%$ de la carga de trabajo de la máquina estudiada se asumió para tratamientos con haz FFF, y en consecuencia los espesores del búnker obedecen a los requerimientos del haz con filtro aplanador. Cuando se aumenta la proporción del haz sin filtro a un $40 \%$, la ventaja de blindaje también lo hace hasta en un $6 \%$, para las primarias, y a 9\%, para las secundarias, logrando que estas distribuciones de carga impacten considerablemente en la reducción de las paredes. Los resultados y porcentajes de disminución de las paredes del búnker se muestran en la tabla 8 .

Como consecuencia de los anteriores resultados, se puede establecer que todo búnker diseñado para linac con haces FF es apto para la operación de un linac con haces FFF. De otra parte, mediante el factor de uso encontrado en este estudio (que corresponde a unidades de tratamiento de un centro de radioterapia moderno), se observa que la ventaja de blindaje al usar haces sin filtración se conserva para los diferentes escenarios analizados; por ejemplo, para el modo FFF se mantiene en el orden del $9 \%$ al 19\% frente al FF. Los resultados de los espesores utilizando los diferentes escenarios con los factores de uso (U) encontrado se presentan en la tabla 9.

Al comparar los resultados de la tabla 9, con los espesores encontrados con factor de uso, $\mathrm{U}$, convencional, se observa que el grosor de las barreras primarias se reduce hasta en un $15 \%$, para haz FF, y hasta en $30 \%$, para haces FFF. Esto indica que, a partir de este tipo de análisis del factor de uso, se puede ofrecer una protección adecuada al público y trabajadores, optimizando de manera significativa los costos en la construcción. Cabe mencionar que los factores de uso encontrados para las barreras primarias fueron sobreestimados de manera significativa en los cálculos; por ejemplo, el factor de uso encontrado para un ángulo de $90^{\circ}$ fue de

Tabla 8. Resultados de requerimientos de blindajes en cm, para haces combinados de $6 \mathrm{MV}$ en proporciones de $80 \% \mathrm{FF}-20 \%$ FFF y $60 \% \mathrm{FF}$ - $40 \% \mathrm{FFF}$

\begin{tabular}{|c|c|c|c|c|c|c|}
\hline Ubicación & Barrera & Espesor (FF) & Espesor (0,8 FF y 0,2 FFF) & Porcentaje de reducción (\%) & Espesor (0,6 FF y 0,4 FFF) & Porcentaje de reducción (\%) \\
\hline $\mathrm{C}$ & Primaria & 151 & 148 & 2 & 144 & 5 \\
\hline A & Secundaria & 79 & 76 & 4 & 72 & 9 \\
\hline $\mathrm{D}$ & Primaria & 159 & 156 & 2 & 152 & 4 \\
\hline B & Secundaria & 95 & 92 & 3 & 88 & 7 \\
\hline G & Primaria & 164 & 161 & 2 & 157 & 4 \\
\hline $\mathrm{H}$ & Secundaria & 94 & 91 & 3 & 88 & 6 \\
\hline $\mathrm{L}$ & Secundaria & 71 & 69 & 3 & 66 & 7 \\
\hline $\mathrm{E}$ & Secundaria & 92 & 89 & 3 & 85 & 8 \\
\hline $\mathrm{J}$ & Secundaria & 81 & 78 & 4 & 74 & 9 \\
\hline
\end{tabular}

Tabla 9. Resultados de requerimientos de blindajes en $\mathrm{cm}$ para haces únicos y combinados utilizando los factores de uso encontrados en este estudio

\begin{tabular}{|c|c|c|c|c|c|}
\hline Ubicación & Barrera & Espesor (FF) & Espesor (FFF) & Espesor (0,8 FF y 0,2 FFF) & Espesor $(0,6 \mathrm{FF}$ y $0,4 \mathrm{FFF})$ \\
\hline $\mathrm{C}$ & Primaria & 128 & 105 & 125 & 121 \\
\hline A & Secundaria & 79 & 69 & 76 & 72 \\
\hline $\mathrm{D}$ & Primaria & 136 & 111 & 133 & 128 \\
\hline B & Secundaria & 95 & 85 & 92 & 88 \\
\hline G & Primaria & 141 & 115 & 138 & 134 \\
\hline $\mathrm{H}$ & Secundaria & 94 & 84 & 91 & 88 \\
\hline $\mathrm{L}$ & Secundaria & 71 & 64 & 69 & 66 \\
\hline $\mathrm{E}$ & Secundaria & 92 & 82 & 89 & 85 \\
\hline $\mathrm{J}$ & Secundaria & 81 & 71 & 78 & 74 \\
\hline
\end{tabular}


Tabla 10. Cantidad de concreto ordinario utilizado para la construcción del búnker tomado como ejemplo

\begin{tabular}{cccc}
\hline Haz & $\mathbf{m}^{\mathbf{3}}$ (concreto) & Haz & $\mathbf{m}^{\mathbf{3}}$ (concreto) \\
\hline FF (U convencional) & 419 & FF (U estudio) & 398 \\
FFF (U convencional) & 363 & FFF (U estudio) & 346 \\
0,8 FF y 0,2 FFF (U convencional) & 395 & 0,8 FF y 0,2 FFF (U estudio) & 374 \\
0,6 FF y 0,4 FFF (U convencional) & 381 & 0,6 FF y 0,4 FFF (U estudio) & 360 \\
\hline
\end{tabular}

0,47\% y se utilizó en el cálculo un $5 \%$, ofreciendo así, mayor seguridad en caso de que se requiera implementar técnicas que contribuyan a un valor mayor. Otro resultado importante de este estudio es que, cuando se utilizan máquinas con dedicación exclusiva a modo FFF y si al mismo tiempo se trabajan factores de uso más realistas como los encontrados, las paredes del búnker pueden ser menos gruesas hasta en un $30 \%$, brindado ventajas significativas en costos y espacio en los diseños de instalaciones.

En la tabla 10 se observa el volumen en metros cúbicos de concreto ordinario necesarios para la construcción del búnker en cada uno de los escenarios estudiados. La disminución del material (concreto ordinario), mediante haces FFF puede llegar hasta un $13 \%$, frente al modo FF (para U convencional), lo cual corresponde a un ahorro en costo de concreto ordinario en el mismo porcentaje. Si se utilizara un factor de uso como el encontrado en este estudio, los ahorros en material de concreto se pueden aumentar hasta un $17 \%$.

Los resultados de las áreas libres dentro del búnker fueron 107 y $113 \mathrm{~m}^{2}$ para modos FF y FFF, respectivamente. De lo anterior, para este ejemplo en específico, se puede concluir que el ahorro en espacio es de $6 \mathrm{~m}^{2}$. Si se utiliza el factor de uso encontrado en este estudio, el ahorro es espacio se incrementa a $11,8 \mathrm{~m}^{2}$. Por consiguiente, la utilización de haces sin filtración brinda ventajas no solo en ahorro en material de construcción, sino también en el área destinada para la construcción de un búnker o amplitud de la sala de tratamiento.

\section{Conclusiones}

Se realizó un estudio de la aplicación de haces de fotones con filtro y sin filtro aplanador, producidos por aceleradores lineales clínicos, con el objetivo de establecer la influencia del filtro en los parámetros usados para el cálculo de blindajes de los búnkeres en los cuales operan. Se analizó, también, la carga de trabajo y el factor de uso de haz primario en un acelerador dedicado a radioterapia con técnicas IMRT o VMAT, y otro en el cual se aplican tratamientos tanto en modalidad 3DCRT como IMRT, con el fin de tener un panorama real correspondiente a una práctica de radioterapia moderna y su efecto en el cálculo de blindajes.

Los resultados de este trabajo corroboran la importancia de conocer la tecnología que se va a adquirir o las actualizaciones tecnológicas que se van a implementar en un centro de radioterapia, y la consecuente necesidad de evaluar la infraestructura física existente o de planear la nueva con una visión muy clara de cómo el servicio puede evolucionar. En efecto, la adquisición de un linac que usa el mismo haz de electrones para crear haces FF y FFF $6 \mathrm{MV}$, puede implicar una diferencia de $15 \%$ en la energía media del haz, lo cual puede conllevar una reducción importante de los blindajes requeridos. Igualmente, la disminución de la carga de fuga inherente a los haces FFF reduce el espesor de los blindajes secundarios.

En este trabajo se analizaron diferentes opciones de combinación de modalidades de tratamiento usando haces FFF y las implicaciones importantes en los costos y en las posibilidades de optimizar el espacio disponible para el búnker. Cuando se utilizan modalidades combinadas, aplicando haces con y sin filtro aplanador, las ventajas de los haces FFF en los blindajes requeridos se ven reducidas, y depende de la combinación utilizada. Si un linac es operado con haces FF y FFF, la evaluación de blindaje deberá tener en cuenta las cargas de trabajo para los dos haces.

Por otra parte, nuestros resultados corroboran la importancia de los análisis técnicos que deben realizarse antes de la introducción de nuevas tecnologías o actualización de las existentes, y del criterio que los físicos médicos deben tener para el apoyo a las directivas de una instalación en la toma de decisiones sobre el diseño. Si en un búnker existente se progresa de técnicas 3DCRT a IMRT con haces FF y FFF, es necesaria una evaluación de la instalación considerando la normativa nacional vigente o recomendaciones internacionales acordes con las nuevas tecnologías. En particular, por ejemplo, la tendencia a emplear cada vez más los haces 
FFF con fracciones de altas dosis, podría afectar significativamente la carga de trabajo clínica anual, especialmente si el acelerador es dedicado a los tratamientos hipofraccionados.

\section{Agradecimientos}

Un agradecimiento muy especial al Grupo de Física Médica del Centro Javeriano de Oncología, en particular al físico médico Alejandro Marín, por su apoyo en la disponibilidad de la información para el análisis de carga de trabajo y factores de uso en la práctica.

\section{Referencias}

[1] Y. Xiao, S. Kry y R. Popple, "Flattening filter-free accelerators: a report from the AAPM therapy emerging technology assessment work group", Journal of Applied Clinical Medical Physics, vol. 16, n. ${ }^{\circ}$ 3, pp. 12-29, 2015. https://doi.org/10.1120/jacmp.v16i3.5219

[2] S. Stevens, K. Rosser y J. Bedford, "A 4 MV flattening filter-free beam: commissioning and application to conformal therapy and volumetric modulated arc therapy", Physics in Medicine and Biology, vol. 56, n. ${ }^{\circ}$ 13, pp. 38093824, 2011.

[3] G. Budgell, K. Brown, J. Cashmore et al., "IPEM topical report 1: guidance on implementing flattening filter free (FFF) radiotherapy", Physics in Medicine \& Biology, vol. 61, n. ${ }^{\circ} 23$, pp. 8360-8394, 2016. https://doi. org/10.1088/0031-9155/61/23/8360

[4] M. Lind, Characteristics of a flattening filter free photon beam-measurements and Monte Carlo simulations, Tesis de doctorado, Department of Radiation Physics, Lund University, Lund, 2008.

[5] O. Vassiliev, "Dosimetric properties of photon beams from a flattening filter free clinical accelerator", Physics in Medicine Biology, vol. 51, n. ${ }^{\circ}$ 7, pp. 1907-1917, 2006. https://doi.org/10.1088/0031-9155/51/7/019

[6] O. Vassiliev, "Monte Carlo study of photon felds from a flattening filter-free clinical accelerator", Medical Physics, vol. 33, n. ${ }^{\circ}$ 4, pp. 820-827, 2006. https://doi. org/10.1118/1.2174720

[7] F. Khan, The Physics of Radiaton Therapy. Wolters-Kluwer, 2014.

[8] D. Sheikh-Bagheri y D. Rogers, "Monte Carlo calculation of nine megavoltage photon beam spectra using the
BEAM code”, Medical Physics, vol. 29, n. ${ }^{\circ}$ 3, pp. 391-402, 2002. https://doi.org/10.1118/1.1445413

[9] National Institute of Standards and Technology (NIST), $X$-ray mass attenuation coefficients, Gaithersburg MD, 2019.

[10] P. LaRiviere y W. Nelson, "Primary and leakage radiation calculations at 6, 10 and $25 \mathrm{MeV}$ ", Medical Physics, vol. 47, n. ${ }^{\circ}$ 6, pp. 811-814, 1984. https://doi.org/10.1097/00004032198412000-00001

[11] D. Trubey, "A survey of empirical functions used to fit gamma-ray buildup factors", Radiation Shielding Information Center, Estados Unidos, ORNL-RSIC-10, feb. 01, 1966.

[12] J. Rodgers, "Analysis of tenth-value-layers for common shielding materials for a robotically mounted stereotactic radiosurgery machine", Medical Physics, vol. 92, n. ${ }^{\circ}$ 4, pp. 379-386, 2007. https://doi.org/10.1097/01.HP.0000252258.58618.6a

[13] National Council on Radiation Protection and Measurements (NCRP), "Structural shielding design and evaluation for megavoltage $\mathrm{X}$ - and gamma ray radiotherapy facilities", Bethesda MD. NCRP Report No. 151, 2005.

[14] S. Kry, R. Mohan, y O. Vassiliev "Treatment vault shielding for a flattening filter-free medical linear accelerator", Physics in Medicine and Biology, vol. 54, n. ${ }^{\circ}$ 5, pp. 12651273, 2009. https://doi.org/10.1088/0031-9155/54/5/011

[15]O. Vassiliev, “Treatment planning study of liver cancer treatments with a flattening filter free linear accelerator", Medical Physics, vol. 33, n. ${ }^{\circ}$ 6, pt. 7, pp. 221, 2006. https://doi.org/10.1118/1.2240996

[16] U. Titt, O. Vassiliev y F. Pönisch, "Monte Carlo study of backscatter in a flattening filter free clinical accelerator", Medical Physics, vol. 33, n. 9 9, pp. 3270-3273, 2006. https://doi.org/10.1118/1.2229430

[17]B. Mishra y T. Palani, "Structural shielding design of a $6 \mathrm{mv}$ flattening filter free linear accelerator: Indian scenario", Medical Physics, vol. 42, n. ${ }^{\circ}$ 1, pp. 18-24, 2017. https://doi.org/10.4103/jmp.JMP_99_16

[18]M. Garau. Radiobiology of stereotactic body radiation therapy (SBRT)", Reports of Practical Oncology and Radiotherapy, vol. 22, n. ${ }^{\circ}$ 2, pp. 86-95, 2017. 10.1016/j. rpor.2017.02.010

[19]J. Cashmore, "The characterization of unflattened photon beams from a $6 \mathrm{MV}$ linear accelerator", Physics in Medicine and Biology, vol. 53, n. ${ }^{\circ}$ 7, pp. 1933-1946, 2008. https://doi.org/10.1088/0031-9155/53/7/009 